\title{
Special Issue in Honor of Masanao Aoki
}

\author{
Yuji Aruka · Mauro Gallegati · Hiroshi Yoshikawa
}

Published online: 17 March 2015

(C) Springer-Verlag Berlin Heidelberg 2015

\section{Preface}

Masanao Aoki (1931, Hiroshima; 1953 BA and 1955 MSc in Physics, the University of Tokyo; 1960 PhD in Engineering, UCLA; Professor of Engineering, 1960-1974 UCLA and Berkley; Professor of Economics, 1974-2002 Illinois and UCLA) is an outstanding scholar in several fields of Economics. He is very special to the JEIC community because he is one of the founder-fathers of the ESHIA and a pioneer of the HIA approach.

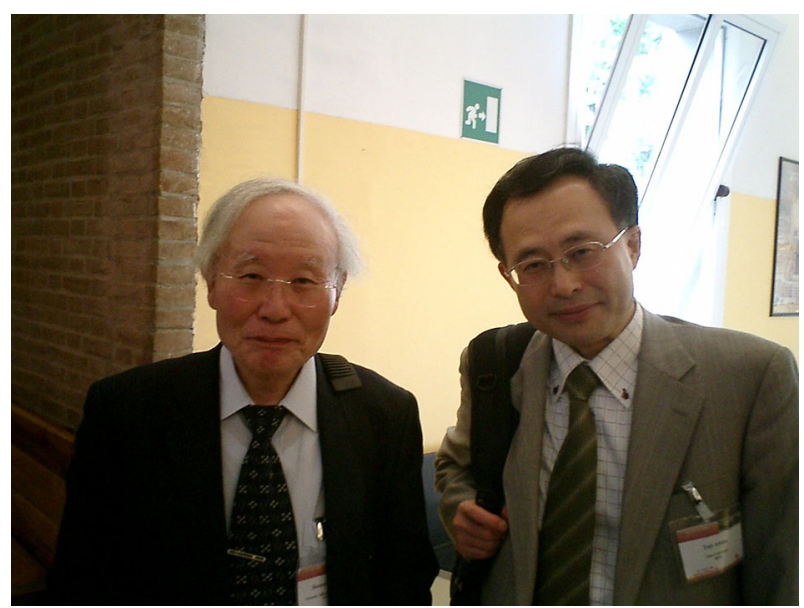

Taken at the conference building of the First Meeting of ESHIA, June 2006, University of Bologna, Italy

Y. Aruka · M. Gallegati $\cdot$ H. Yoshikawa $(\varangle)$

Tokyo, Japan

e-mail: yoshikawa@e.u-tokyo.ac.jp 
His contributions to Economics could be classified into the following five topics:

- The dual and adaptive theory of control and the applications in dynamic programming, where the idea of stochastic approximation is applied to economic modeling (On Sufficient Conditions for Optimal Stabilization Policies, Review of Economic Studies, vol. 40(1), 1973; Approximation scheme for evaluating some terminal capital stock, Journal of Economic Theory, vol. 6(3), 1973).

- The control theory and parameters estimation of large-scaled and decentralized system, where he successfully introduces the controllability and the related ideas in the system theory into the macroeconomic policy (On a Generalization of Tinbergen's Condition in the Theory of Policy to Dynamic Models, Review of Economic Studies, vol. 42(2), 1975; Local Controllability of a Decentralized Economic System, Review of Economic Studies, vol. 41(1), 1974; Non-interacting control of macroeconomic variables: Implications on policy mix considerations, Journal of Econometrics, vol. 2(3), 1974);

- The applications of control theory and system theory to the economic system as well as the neoclassical economic models (Optimal control and system theory in dynamic economic analysis, North-Holland, 1976; Dynamic analysis of open economies, Academic Press, 1982).

- The development of a new algorithm for the time series model and the applications of them to the economic data where the models were also featured by Aoki's method on aggregation in a dynamic system, which virtually happened to be the same method "co-integration" developed by C. Granger (Note on economic time series analysis: system theoretic perspective, Springer Verlag, 1982; State space modeling of time series, Springer Verlag, 1997; State space modeling of time series, Springer Verlag, 2002). At this stage he argues that Instrumental Variable Method is not only precisely argued in the context of stochastic realization due to Lindquint and Picci but also is developed completely as a software package. This method can be applied to the multivariable time series. These works were highly appreciated and obtained a good popularity. He thus had invited lectures in the American Statistical Association and International Forecasting Society. Econometric Review also arranged the special issue on this subject in 1991. Amid an abundance of ARMA, the models of innovation could be estimated by the state space modeling. This algorithm was commercialized also as a software package for sales.

- The 5th stage is the most exciting stage to construct a new perspective for economic science in line with the Society for Economic Science for Heterogeneous Interacting Agents he co-founded in 2006. This is represented by the three books: New approaches to macroeconomic modeling: evolutionary stochastic dynamics, multiple equilibria, and externalities as field effects. Cambridge University Press, New York, 1996; Modeling aggregate behavior and fluctuations in economics: stochastic views of interacting agents, Cambridge University Press, New York, 2002; (with H. Yoshikawa) Reconstructing macroeconomics: a perspective from statistical physics and combinatorial stochastic processes, Cambridge University Press, Cambridge, New York, 2006. The new method is featured by Statistical Physics and Combinatorial Stochastic Processes. Equilibria is not treated as a fixed 
points. The system may be subject to non-self averaging and mutant could emerge internally. These studies can rightly establish a set of theoretical foundations to socio-econophysics.

Masanao proposes a new, alternative approach to economics, based on the statistical physics. For the mainstream economist this comes as an eresia. He still believes in determinism, equilibrium and reductionism (as Newton did). Equilibrium in particular is considered an aberration for a statistical physicist. The macro behavior of a system does not reflect those of its constituents. In economics it means that a, e.g., a sector can be in equilibrium even if some firm overproduces the good and other underproduces it. So there can be disequilibrium at the individual level, but equilibrium at the aggregate one. Equilibrium thus becomes a statistically distributed concept. Aoki suggests this can be studied in Economics with the Master Equation approach, which, at the same time, overcomes the Representative Agent approach and provides a proper tool for analyzing a system with many heterogeneous interacting agents.

It is worth noting that the modern micro-founded macroeconomic theory actually emphasizes heterogeneity. However, the Lucas rational expectations theory, the Mortensen search model, and DSGE (Dynamic Stochastic General Equilibrium) models all presume one distribution of variable of interest common to all micro agents in model. As such, they effectively presume the representative agent whose optimization is analyzed in detail. Heterogeneity in standard models is not "true" heterogeneity. Masanao's breakthrough works have opened the door to analyzing true heterogeneity in economic models.

This issue of the JEIC is dedicated and honoring Masanao. Some of the contributors are personal friends to him, most of them are his followers, all of them love him.

The list of papers can be divided into 3 categories.

A. Macroeconomics

1. Sorin Solomon, Natasa Golo: Microeconomic Structure determines Macroeconomic Dynamics; Aoki defeats the Representative Agent

2. Hiroshi Yoshikawa: Stochastic Macro-equilibrium: A Microfoundation for the Keynesian Economics

3. Hideaki Aoyama, Hiroshi Iyetomi, Hiroshi Yoshikawa: Equilibrium Distribution of Labor Productivity: A Theoretical Model

4. Yoshi Fujiwara, Hideaki Aoyama, Mauro Gallegati: Micro-Macro Relation of Production: Double Scaling Law for Statistical Physics of Economy

5. Enrico Scalas, Tijana Radivojevic', Ubaldo Garibaldi: Wealth distribution and the Lorenz curve: A finitary approach

B. Agent-based modeling

5. Simone Landini, Mauro Gallegati, Joseph Stiglitz: Economies with Heterogeneous Interacting Learning Agents

6. Daniel Fricke, Thomas Lux: The Effects of a Financial Transaction Tax in an Artificial Financial Market 
C. Macroeconomic policy

7. Raymond Hawkins: Okun's Law and Anelastic Relaxation in Economics

8. Willi Semmler, Christian R. Proano, Matthieu Charpe, Peter Flaschel, Hans-Martin Krolzig, Daniele Tavani: Credit-Driven Investment, Heterogeneous Labor Markets and Macroeconomic Dynamics

9. Raymond Hawkins, Jeffrey Speakes, Daniel Hamilton: Monetary Policy and PID Control

All the papers aim to move small steps toward a new economic theory by following Masanao's approach. Readers will judge if they reached this goal. Now, let's enjoy Masanao's pathbreaking ideas. 